\title{
Multi-objective Model-free Control based on Population Dynamics and Cooperative Games
}

\author{
J. Barreiro-Gomez, C. Ocampo-Martinez, Senior Member, IEEE, J.M. Maestre and \\ N. Quijano, Senior Member, IEEE
}

\begin{abstract}
This work solves a model-free resource allocation problem with two objectives. These objectives represent both cooperation and competition directions. It is proposed a solution that combines a centralized cooperative game approach using the Shapley value to determine a proper partitioning of the system, and a decentralized non-cooperative game approach using the Nash equilibrium to achieve the control objective by means of both partial and local information. Furthermore, invariant set and stability analysis are discussed for the noncooperative game approach. Another contribution regarding the cooperative game approach relies on a novel and alternative way to compute the Shapley value for the chosen characteristic function. This alternative computational way is proposed in order to mitigate the commonly high computational burden issue, which is associated to the combinatorial explosion associated to the cooperative game approach.
\end{abstract}

Index Terms-Population dynamics, Nash equilibrium, cooperative games, Shapley value, resource allocation, partitioning approach.

\section{INTRODUCTION}

Game theory has become a powerful tool in the design of learning and optimization-based systems. Both cooperative and non-cooperative game approaches have been widely used in the design of controllers depending on the control objective. In some cases, it is more suitable to work with a cooperative perspective when agents can collaborate among them, whereas there are other situations in which it is more appropriate to set the problem as a competition. However, there are control problems that integrate cooperation and competition in two different stages. The ideal centralized control scheme in which a single entity governs a system using full information, disposing enough time to gather all the measurements, and to compute and transmit all the control actions, is not quite common.

J. Barreiro-Gomez and C. Ocampo-Martinez are with the Automatic Control Department, Universitat Politècnica de Catalunya, Institut de Robòtica i Informàtica Industrial (CSIC-UPC), Llorens i Artigas, 4-6, 08028 Barcelona, Spain. \{jbarreiro, cocampo\}@iri.upc.edu

J. Barreiro-Gomez and N. Quijano are with Departamento de Ingeniería Eléctrica y Electrónica, Universidad de los Andes, Carrera $1^{\mathrm{A}}$ No $18 \mathrm{~A}-10$, Colombia \{j.barreiro135, nquijano\}@uniandes.edu.co.

J.M. Maestre is with the Departamento de Ingeniería de Sistemas y Automática, Universidad de Sevilla, Sevilla 41092, Spain, pepemaestre@us.es.

Authors would like to thank COLCIENCIAS (doctorado nacional grant 6172) and Agència de Gestió d'Ajust Universitaris i de Recerca AGAUR for supporting J. Barreiro-Gomez. Authors also would like to thank Mexichem, Colombia for partially supporting this research through the project "Drenaje urbano y cambio climtico: hacia los sistemas de alcantarillado del futuro. Fase II", the projects ECOCIS (Ref. DPI2013-48243-C2-1-R) and "Control Predictivo en Red" (Ref. DPI2008-05818) from the Spanish Ministry of Science and Education, and the European project FP7-ICT DYMASOS (under grant agreement 611281), which have partially supported this work.
This work focuses on one of the recent trends in the development of non-centralized control techniques. In particular, during the last years different control strategies have been proposed to create control systems able to adapt dynamically to the evolution of the system and its structure. For example, in [12] plug and play network model predictive controllers are studied. In [3], a method to design simultaneously the communication topology and feedback control laws is proposed. Likewise in [6], a control method that switches between different linear feedback controllers to attain a trade-off between communication burden and performance is proposed. The rationale of this work is somehow similar since the control architecture changes its topology in order to reduce the communication burden. However, unlike the aforementioned works, the control strategy proposed in this paper uses both a cooperative and a non-cooperative gametheory approach. Furthermore, the proposed approach is a model-free control inspired by the resource allocation problem.

Another contribution of this paper is to deepen into the relationship between game theory and control. Being the mathematical field that deals with situations of mutual interaction [8], game theory has a natural application specifically in the context of non-centralized controllers. Broadly speaking, game theory has two main branches depending on whether the players of the game are capable to cooperate each other or not. In particular, non-cooperative game theory deals with problems where players make decisions in order to maximize their utilities. One solution of a non-cooperative game is given by the Nash equilibrium, condition in which no player can improve their utilities without the detriment of other players utilities. In contrast, cooperative game theory studies the conditions and payoff rules for groups of players that form coalitions. One possible solution of a cooperative game is given by the Shapley value, which assigns a fair payoff to each player according to what it contributes to the game. There are many control solutions that use both game theory approaches. For instance in [5], an application of noncooperative game theory can be found where a distributed control strategy based on the convergence to a Nash equilibrium is proposed [9]. Furthermore, convergence to Nash equilibrium by using evolutionary game theory has been used in the design of control and optimization strategies [10], [1], [15]. On the other hand, cooperative game theory has been used for example in [6]. In this and other related works such as [7], the links that compose the network topology are transformed into the players of a game and the payoff given 
by the Shapley value [14] is used as a mean to determine their relevance. Other works that mainly use the Shapley value are [4], [2].

In this work, a combination of these two game-theoretical approaches is used to propose a decentralized control strategy. More specifically, a network system is divided into different partitions. These partitions are determined by a criterion based on a cooperative game, i.e., by using the Shapley value. Furthermore, a population-dynamics approach is implemented at each partition. Then, a model-free resource allocation problem is solved at each sub-system by converging to a Nash equilibrium. Moreover, this work proposes a different way to compute the Shapley value for the selected characteristic function in the cooperative game in order to reduce the computational burden, which is one of the main issues when using the game-theoretical approach. Finally, in order to show the performance of the proposed methodology based on cooperative and non-cooperative games, a resource allocation problem in a water system treated in [11] is presented.

The remainder of this paper is organized as follows. Section II presents the population dynamics approach used to find the solution given by the Nash equilibrium. Section III shows the role of the cooperative-game approach in the control problem by finding the solution given by the Shapley value. This section also presents the partitioning criterion based on the Shapley value. Section IV describes the case study and the control objectives. Afterwards, concluding remarks are presented in Section VI.

Notation: Column vectors are denoted by bold style, e.g., x. Scalar numbers are denoted by non-bold style, e.g., $N$. Sets are denoted by calligraphic upper case, e.g., $\mathcal{T}$. Subindex refers to the topologies in the system, and super-index refers to partitions, e.g., $\mathcal{S}_{i}^{j}$ refers to a set of a partition $j$ of a topology $i$. It is worth to point out that super-index is not operational, i.e, $N_{i}^{3}$ refers to partition 3 , and $N_{i}^{3} \neq N_{i} N_{i} N_{i}$. Real numbers are denoted by $\mathbb{R}$, and all the non-negative real numbers by $\mathbb{R}_{+}$. Finally $\mathbb{1}_{N}$ is a column tile with $N$ unitary entries, and the cardinality of set is defined by $|\cdot|$, e.g., $|\mathcal{O}|$ is the number of elements of the set $\mathcal{O}$.

\section{Population Dynamics Approach}

Consider a population composed by a finite and large number of rational agents. In the population evolution, agents make the decision to select among $N$ possible strategies from the set $\mathcal{S}=\{1, \ldots, N\}$, in order to improve their payoff. The population has $T$ possible different topologies that determine how the population is distributed over a certain graph. The set of the population topologies is denoted by $\mathcal{T}=\{1, \ldots, T\}$. Each topology is given by a non-complete graph denoted by $\mathcal{G}_{i}=\left(\mathcal{V}_{i}, \mathcal{E}_{i}, \mathcal{A}_{i}\right)$, where $\mathcal{V}_{i}$ is the set of $N$ nodes representing the strategies $\mathcal{S}, \mathcal{E}_{i}$ is the set of links that represent the possible interaction among agents selecting the corresponding strategies, and $\mathcal{A}_{i}$ is the adjacency matrix. Moreover, each topology $i \in \mathcal{T}$ has $P_{i}$ disjoint partitions. The set of partitions of the population topology $i \in \mathcal{T}$ is given by $\mathcal{P}_{i}=\left\{1, \ldots, P_{i}\right\}$.
The partition $j \in \mathcal{P}_{i}$ of the topology $i \in \mathcal{T}$ is denoted by a complete graph $\mathcal{G}_{i}^{j}=\left(\mathcal{V}_{i}^{j}, \mathcal{E}_{i}^{j}\right)$, where $\mathcal{V}_{i}^{j}$ is the set of $N_{i}^{j}$ nodes representing the set of strategies within the corresponding partition $\mathcal{S}_{i}^{j}$, and $\mathcal{E}_{i}^{j}$ is the set of $N_{i}^{j}\left(N_{i}^{j}-1\right) / 2$ links representing the full information sharing and interaction within each partition. Furthermore, it must be satisfied that all the partitions form the topology, i.e., $\cup_{j \in \mathcal{P}_{i}} \mathcal{G}_{i}^{j}=\mathcal{G}_{i}$, for all $i \in \mathcal{T}$. Also, it is necessary that there are no nodes belonging to more than one partition, i.e., $\cap_{j \in \mathcal{P}_{i}} \mathcal{V}_{i}^{j}=\emptyset$, for all $i \in \mathcal{T}$.

In the population, $x_{l} \in \mathbb{R}_{+}$is the proportion of agents selecting the strategy $l \in \mathcal{S}$. The vector $\mathbf{x} \in \mathbb{R}_{+}^{N}$ is a population state or a strategic distribution composed by all the proportion of agents selecting the available strategies. The set of all the possible population states is given by a simplex denoted by $\Delta=\left\{\mathbf{x} \in \mathbb{R}_{+}^{N}: \mathbf{x}^{\top} \mathbb{1}_{N}=1\right\}$. Similarly, $x_{i, a}^{j} \in \mathbb{R}_{+}$is the proportion of agents selecting the strategy $a \in \mathcal{S}_{i}^{j}$ available in the partition $j \in \mathcal{P}_{i}$ of the topology $i \in \mathcal{T}$. The vector $\mathbf{x}_{i}^{j} \in \mathbb{R}_{+}^{N_{i}^{j}}$ is the strategic distribution of agents in the respective partition and topology. Finally, let $m_{i}^{j}$ be the total mass in the partition given by $m_{i}^{j}=\mathbf{x}_{i}^{j \top} \mathbb{1}_{N_{i}^{j}}$.

The payoff that agents receive for being selecting a particular strategy is given by a function that takes the population state and returns a reward, i.e., fitness functions have a mapping $f_{l}: \Delta \mapsto \mathbb{R}, l \in \mathcal{S}$, and the vector of fitness functions in the population denoted by $\mathbf{F}$ is composed by all the fitness functions $f_{l}(\mathbf{x}), l \in \mathcal{S}$. Similarly, the vector of fitness functions of the partition $j \in \mathcal{P}_{i}$ of the topology $i \in \mathcal{T}$ is denoted by $\mathbf{F}_{i}^{j}$, and it is composed by all the fitness functions $f_{l}(\mathbf{x}), l \in \mathcal{S}_{i}^{j}$. The average function in the population is given by $\bar{f}=\mathbf{x}^{\top} \mathbf{F}$. The average for a partition is given by $\bar{f}_{i}^{j}=\left(\mathbf{x}_{i}^{j^{\top}} \mathbf{F}_{i}^{j}\right) / m_{i}^{j}, j \in \mathcal{P}_{i}, i \in \mathcal{T}$, and $\overline{\mathbf{F}}_{i}^{j}=\mathbb{1}_{N_{i}^{j}} \bar{f}_{i}^{j}$.

The framework of the method proposed in this paper is given by stable games, which establish a condition over fitness functions selection for control design, i.e., $(\mathbf{w}-\mathbf{x})^{\top}(\mathbf{F}(\mathbf{w})-\mathbf{F}(\mathbf{x})) \leq 0, \quad$ for all $\mathbf{x}, \mathbf{w} \in \Delta$.

Replicator Dynamics: The replicator dynamics is one of the six fundamental population dynamics and has been largely used in the solution of engineering problems [13]. These dynamics require information about all elements within the population and it is the reason why it has been considered that partitions at each topology are complete graphs.

For a fixed topology, there is a replicator dynamics system for each partition. For a topology $i \in \mathcal{T}$ and a partition $j \in \mathcal{P}_{i}$, the replicator dynamics are given by

$$
\dot{\mathbf{x}}_{i}^{j}=\operatorname{diag}\left(\mathbf{x}_{i}^{j}\right)\left(\mathbf{F}_{i}^{j}-\overline{\mathbf{F}}_{i}^{j}\right) .
$$

Then, the system switches among topologies in order to use at each iteration instant a limited number of communication links. The equilibrium of interest in (1) for this work is the mixed-strategy Nash equilibrium given by the condition $\mathbf{F}_{i}^{j}=\overline{\mathbf{F}}_{i}^{j}$, for all $j \in \mathcal{P}_{i}, i \in \mathcal{T}$. Due to the fact the partition of a topology is a non-connected graph, this equilibrium is achieved at each partition. Moreover, 
since topologies and partitions are varying over time, it is necessary to identify the equilibrium for all topologies and partition, i.e., $f_{k}(\mathbf{x})=f_{l}(\mathbf{x})$, for all $k, l \in \mathcal{S}$.

Now, it is shown that each partition satisfies an invariant set and consequently the whole set $\Delta$ is invariant under the replicator dynamics (1) and switching partitioned topologies.

Proposition 1: If initial condition $\mathrm{x}_{0} \in \Delta$, then $\mathrm{x} \in$ $\Delta, \forall t \geq 0$, i.e., the simplex $\Delta$ is an invariant set under replicator equation (1) and switching partitioned topologies.

Proof: In order to proof that $\Delta$ is an invariant set, it is shown that the sum for all partition and topologies of $\dot{\mathbf{x}}_{i}^{j}$ for all $i \in \mathcal{T}$ and $j \in \mathcal{P}_{i}$ is null ${ }^{1}$, i.e.,

$$
\begin{aligned}
\sum_{i \in \mathcal{T}} \sum_{j \in \mathcal{P}_{i}} \mathbb{1}_{N_{i}^{j}}^{\top} \dot{\mathbf{x}}_{i}^{j} & =\sum_{i \in \mathcal{T}} \sum_{j \in \mathcal{P}_{i}} \mathbf{x}_{i}^{j^{\top}}\left(\mathbf{F}_{i}^{j}-\frac{1}{m_{i}^{j}} \mathbb{1}_{N_{i}^{j}} \mathbf{x}_{i}^{j^{\top}} \mathbf{F}_{i}^{j}\right) \\
& =\sum_{i \in \mathcal{T}} \sum_{j \in \mathcal{P}_{i}}\left(\mathbf{x}_{i}^{j^{\top}} \mathbf{F}_{i}^{j}-\mathbf{x}_{i}^{j^{\top}} \mathbf{F}_{i}^{j}\right) \\
& =0
\end{aligned}
$$

which completes the proof.

Proposition 2: Let $\mathbf{F}$ be a stable game, then the switched system (1) is uniformly stable since it admits a common Lyapunov function. candidate $^{1}$

Proof: Consider the entropy function as a Lyapunov

$$
\begin{aligned}
L(\mathbf{x}) & =-\sum_{i \in \mathcal{T}} \sum_{j \in \mathcal{P}_{i}} \sum_{a \in \mathcal{S}_{i}^{j}} x_{i, a}^{j}{ }^{*} \ln \left(\frac{x_{i, a}^{j}}{x_{i, a}^{j}{ }^{*}}\right), \\
& =-\sum_{i \in \mathcal{T}} \sum_{l \in \mathcal{S}} x_{l}{ }^{*} \ln \left(\frac{x_{l}}{x_{l}{ }^{*}}\right) .
\end{aligned}
$$

This is a valid Lyapunov function since $L\left(\mathrm{x}^{*}\right)=0$ and $L(\mathbf{x})>0$, for all $\mathbf{x} \neq \mathbf{x}^{*}$, this is checked by using the Jensen's inequality (i.e., $E(f(x)) \geq f(E(x))$ for any convex function as the logarithm). Its derivative is

$$
\dot{L}(\mathbf{x})=-\sum_{i \in \mathcal{T}} \sum_{j \in \mathcal{P}_{i}} \sum_{a \in \mathcal{S}_{i}^{j}} \frac{x_{i, a}^{j}{ }^{*}}{x_{i, a}^{j}} \dot{x}_{i, a}^{j} .
$$

Replacing dynamics in the Lyapunov function derivative,

$$
\begin{aligned}
\dot{L}(\mathbf{x}) & =-\sum_{i \in \mathcal{T}} \sum_{j \in \mathcal{P}_{i}} \mathbf{x}_{i}^{j^{* \top}}\left(\mathbf{F}_{i}^{j}-\frac{1}{m_{i}^{j}} \mathbb{1}_{N_{i}^{j}} \mathbf{x}_{i}^{j^{\top}} \mathbf{F}_{i}^{j}\right) \\
& =-\sum_{i \in \mathcal{T}} \sum_{j \in \mathcal{P}_{i}}\left(\mathbf{x}_{i}^{j^{*}}-\mathbf{x}_{i}^{j}\right)^{\top} \mathbf{F}_{i}^{j} \\
& \leq 0
\end{aligned}
$$

and equality holds when $\mathbf{x}_{i}^{j^{*}}=\mathbf{x}_{i}^{j}$, for all $i \in \mathcal{T}$, and $j \in \mathcal{P}_{i}$. The partitions for all the topologies admit the same Lyapunov function, then the system is uniformly stable for any convex combination of topologies.

\footnotetext{
${ }^{1}$ Details of this proof have been omitted due to the lack of space.
}

\section{Cooperative Game Role}

Cooperative game theory studies how players can be grouped into coalitions in order to achieve a common goal and how to share the costs or benefits derived from their joint effort. In this work it is proposed to use this theory to form coalitions within the control system. Different topologies are considered -and consequently different partitions- in the system, which may vary over time in order to achieve an equilibrium with a reduction of the communication burden. To this end, a cooperative game with transferable utility is defined as a pair $(\mathcal{V}, V)$, where $\mathcal{V}=\{1, . ., N\}$ is the set of players, and $V$ is the characteristic function. From the cooperative game theoretical point of view, for each topology $i \in \mathcal{T}$, each node $l \in \mathcal{V}_{i}$ is considered as a player, and each partition $j \in \mathcal{P}_{i}$ represents a coalition of players.

The characteristic function denoted by $V$ takes a coalition from the power set $2^{\mathcal{V}}$ and returns a real value. Formally, the characteristic function is a mapping $V: 2^{\mathcal{V}} \mapsto \mathbb{R}$. For each coalition $\mathcal{O} \subseteq \mathcal{V}, V(\mathcal{O})$ is the value that the players can share among themselves. Additionally, for the empty coalition, $V(\emptyset) \triangleq 0$.

A solution of the cooperative game is an allocation rule that gives a division of the value of the coalition among its members in a fair way, i.e., achieving a condition in which each player receives a benefit according to its contribution to the coalition. Let $\mathbf{y} \in \mathbb{R}^{N}$ be the payoff vector given by $\mathbf{y}=\left[\begin{array}{lll}y_{1} & \cdots & y_{N}\end{array}\right]^{\top}$. Some desirable properties for the distribution of the $V(\mathcal{O})$ among the players are: 1) Efficiency: $\sum_{l \in \mathcal{O}} y_{l} \leq V(\mathcal{V})$; 2) Coalitional rationality: $\sum_{l \in \mathcal{O}} y_{l}=V(\mathcal{O})$ for all coalitions $\mathcal{O} \subseteq \mathcal{V}$; 3) Individual rationality: $y_{l} \geq V(\{l\})$, for all $l \in \mathcal{V}$. Prior to defining the characteristic function, the costs associated to each coalition are defined as follows:

$$
C(\mathcal{O})=\frac{1}{|\mathcal{O}|} \sum_{l \in \mathcal{O}} C_{l}
$$

where $C_{l}$ is the individual cost of player $l \in \mathcal{V}$. Afterwards, the characteristic function is given by the difference between the sum of individual costs and the cost of the corresponding coalition. Then, it is characterized the difference when adopting a coalition, i.e.,

$$
V(\mathcal{O})=\sum_{l \in \mathcal{O}} C_{l}-C(\mathcal{O})
$$

A payoff rule that satisfies the mentioned desired requirements is the Shapley value for all the players $l \in \mathcal{V}$ [14], which is given by

$$
\Phi(l)=\sum_{\mathcal{O} \subseteq \mathcal{V} \backslash\{l\}} \frac{|\mathcal{O}| !(N-|\mathcal{O}|-1) !}{N !}(V(\mathcal{O} \cup\{l\})-V(\mathcal{O})) .
$$

Notice that the sum considers all the possible coalitions to which player $l \in \mathcal{V}$ can be added. One of the main issues of working with cooperative games is the high computational burden in the computation of the Shapley value for all the players when using (4). In particular, the combinatorial explosion when the number of players is high is a common 
issue in this context. Once the characteristic function has been defined as (3), a mathematical relation between the Shapley values can be determined in order to mitigate the high computational cost. This result is shown by using Proposition 3 stated next.

Proposition 3: Let (3) be the characteristic function of a cooperative game with the set of players $\mathcal{V}=\{1, \ldots, N\}$. Let $C_{l}$ be the cost associated to each player $l \in \mathcal{V}$, and (2) be the costs associated to each coalition $\mathcal{O} \subseteq \mathcal{V}$. If one Shapley value $\Phi(l)$ for any player $l \in \mathcal{V}$ is known, then all the Shapley values for all the players can be found by using a low-computational-cost operation of the form $\Phi(r)=\Phi(l)+\left(C_{r}-C_{l}\right) \Theta$, where $r \in \mathcal{V}$ is any other player, and $\Theta$ is a constant for the cooperative game whose value only depends on the number of players $N$.

Proof: In order to simplify the notation, it is defined the operator $\Psi(\mathcal{O})=(|\mathcal{O}| !(N-|\mathcal{O}|-1) !) /(N !)$. Then, the Shapley value $\Phi(l)$ of the player $l \in \mathcal{V}$ in (4) may be rewritten by expressing the set of coalitions to which the player $l \in \mathcal{V}$ can be added, in terms of a second player $r \in \mathcal{V}$ as follows ${ }^{2}$ :

$$
\begin{aligned}
\Phi(l) & =\sum_{\mathcal{O} \subseteq \mathcal{V} \backslash\{l, r\}} \Psi(\mathcal{O})(V(\mathcal{O} \cup\{l\})-V(\mathcal{O}))+ \\
& \sum_{\mathcal{O} \subseteq \mathcal{V} \backslash\{l, r\}} \Psi(\mathcal{O} \cup\{r\})(V(\mathcal{O} \cup\{r\} \cup\{l\})-V(\mathcal{O} \cup\{r\})) .
\end{aligned}
$$

Similarly, the Shapley value $\Phi(r)$ of the player $r \in \mathcal{V}$ may be written in terms of $l \in \mathcal{V}$. Then, it is found the difference between the Shapley values $\Phi(r)$, and $\Phi(l)$, denoted by $\tilde{\Phi}(r, l)=\Phi(r)-\Phi(l)$. Also, replacing (3) and (2), and taking into account that $\Psi(\mathcal{O} \cup\{r\})=\Psi(\mathcal{O} \cup\{l\})$, i.e.,

$$
\begin{aligned}
\tilde{\Phi}(r, l)= & \sum_{\mathcal{O} \subseteq \mathcal{V} \backslash\{l, r\}} \Psi(\mathcal{O})\left\{\left(1-\frac{1}{|\mathcal{O}|+1}\right)\left(C_{r}-C_{l}\right)\right\}+ \\
& \sum_{\mathcal{O} \subseteq \mathcal{V} \backslash\{l, r\}} \Psi(\mathcal{O} \cup\{r\})\left\{\left(1-\frac{1}{|\mathcal{O}|+1}\right)\left(C_{r}-C_{l}\right)\right\} .
\end{aligned}
$$

Briefly, the difference between the Shapley values $\tilde{\Phi}(r, l)=\Phi(r)-\Phi(l)$ is given by

$$
\tilde{\Phi}(r, l)=(C_{r}-\underbrace{\left.C_{l}\right) \underbrace{}_{\mathcal{O} \subseteq \mathcal{V} \backslash\{l, r\}} \overbrace{(\Psi(\mathcal{O})+\Psi(\mathcal{O} \cup\{r\}))}^{\theta_{1}} \overbrace{\left(\frac{|\mathcal{O}|}{|\mathcal{O}|+1}\right)}^{\theta_{2}}}_{\Theta},
$$

the constant value $\Theta$ can be re-written as

$$
\begin{aligned}
& \Theta= \sum_{s=1}^{N-2}\{\overbrace{\left(\frac{(N-2) !}{s !(N-2-s) !}\right)}^{\theta_{3}} \overbrace{\left(\frac{s}{s+1}\right)}^{\theta_{2}} \\
&\underbrace{\left(\frac{s !(N-s-1) !+(s+1) !(N-s-2) !}{N !}\right)}_{\theta_{1}}\},
\end{aligned}
$$

\begin{tabular}{|c|c|c|}
\hline $\begin{array}{c}\text { Total Number } \\
\text { of players } \\
N\end{array}$ & $\begin{array}{c}\text { Computational } \\
\text { burden using (4) } \\
\text { Time [s] }\end{array}$ & $\begin{array}{c}\text { Computational } \\
\text { burden using (5) } \\
\text { Time [s] }\end{array}$ \\
\hline 3 & $\overline{0.4232}$ & $\overline{0.1461}$ \\
\hline 4 & 0.8020 & 0.1912 \\
\hline 10 & 5.9998 & 0.6120 \\
\hline 14 & 110.6065 & 7.9103 \\
\hline 18 & 53938.9433 & 2996.6542 \\
\hline
\end{tabular}

${ }^{2}$ Details of this proof have been omitted due to the lack of space.
TABLE I

COMPARISON OF COMPUTATIONAL BURDEN FOR COMPUTING THE

the factor $\theta_{3}$ represents the amount of coalitions that can be formed in the cooperative game with $s$ players, i.e., $|\mathcal{O}|=s$. Finally $\Phi(r)=\Phi(l)+\left(C_{r}-C_{l}\right) \Theta$, obtaining the desired relationship that completes the proof.

In order to verify the difference between the computational burden of computing the Shapley value with (4), and by using the relation proposed in Proposition 3 with the constant value (5), different Shapley values for several amount of players have been computed, and the respective computational burden is presented in Table I.

\section{Partitioning Procedure}

In order to perform the partitioning of the system, first it is defined a time $\Delta t$ that determines when the proper topology is evaluated. Every time $k \in \mathbb{Z}_{+}$with the sampling time $\Delta t$, the Shapley value $\Phi(l, k)$ of all the players $l \in \mathcal{V}$ is computed by using the low-computational-cost operation using the factor $\Theta$ in (5). As a second necessary parameter to define is the size of the desired coalitions denoted by $g$, i.e., perform partitions of $g$ number of players.

Remark 1: Notice that depending on the desired size of the coalitions, it is possible to obtain at most one coalition composed by a number of players lower than $g$, e.g., consider the case of $N=8$ players, and if it is desired to perform a partition of size $g=3$, then there are going to be obtained two coalitions of $g$ players and one coalition of $2<g$ players.

In the partitioning procedure, it is desired to gather the player with the highest Shapley value with the $g-1$ players with the lowest Shapley values. In this sense, it is possible to make a cooperation in which the best players share their benefits with those in worse situation. It is worth to highlight that this partitioning criterion might be different depending on the control objectives and the system dynamical behavior, but it is proper to make this partitioning in function of the Shapley value.

\section{CAse Study}

The case study is shown in Figure 1a), which is composed by $N=12$ tanks. There are two control objectives. The first control objective is to maintain all the tanks at the same and maximum level; this objective is solved by finding a Nash equilibrium. Furthermore, the costs associated to the communication links determine a Key Performance Indicator (KPI) given by $K P I_{\text {links }}=\frac{1}{2} \int_{t_{0}}^{t_{f}} \mathbb{1}_{N}^{\top} \mathcal{A}_{i} \mathbb{1}_{N} d t, i \in \mathcal{T}$, for the current topology $i \in \mathcal{T}$, where $t_{0}$ is an initial time and $t_{f}$ a final time. As a second control objective, it is desired to determine the fair cost that each player should pay for these 
communication links according to the player contribution to the system in order to achieve the desired equilibrium. Then, the fair cost that each player should pay is expressed in function of the Shapley value, i.e.,

$$
K P I_{\text {links }, l}=\frac{\Phi(l, k)}{\sum_{r=1}^{N} \Phi(r, k)} K P I_{\text {links }}, l \in \mathcal{V} .
$$

The Shapley value is normalized in (6) since it is computed in terms of the error rather than economical units. These communication links share, in a local way, information about the measured levels in order to achieve the first control objective. Notice that this is a decentralized control scheme since each control action is computed by using only partial and local information.

In order to solve the control problem, it is proposed to perform a partitioning based on the Shapley value as presented in Section III. Therefore, the costs associated to each player are going to be the error between the current level and the maximum level capacity of each tank, i.e.,

$$
C_{l}=h_{\max , l}-h_{l},
$$

where $h_{\max , l}$ denotes the maximum possible level for the $l^{\text {th }}$ tank. This cost characterization shows that it is more costly to increase the level in a tank rather than reduce it. Once the partitions are made, a population-dynamics approach is applied to each partition where the fitness function for each strategy is given by the error $f_{l}=h_{\max , l}-h_{l}$. Notice that this is a model-free approach since knowledge about dynamics is not required in the design, i.e., $f_{l}$ is computed just by measuring $h_{l}$. It is assumed that this fitness function is always non-negative since physically the current measured level $h_{l} \leq h_{\max , l}$. Notice that, with this fitness function, more resource is assigned to empty tanks. Moreover, this fitness function is decreasing with respect to $x_{i}$, since the error is expected to be reduced when more resource is assigned to the tank. In this way, it is guaranteed that the population game $\mathbf{F}$ is stable and then Proposition 2 holds.

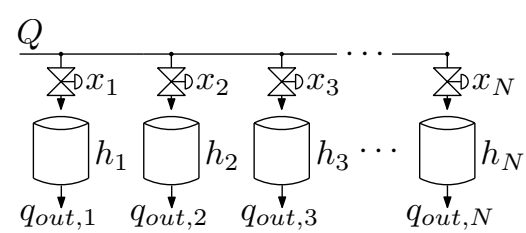

a)

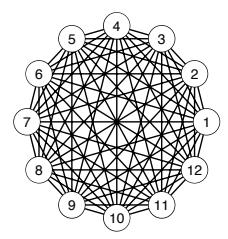

b)
Fig. 1. a) Case study: Resource $Q$ allocation throughout $N$ tanks. b) Complete graph given by the grand coalition.

The dynamics for the $l^{\text {th }}$ tank in continuous time are given by $\dot{h}_{l}=q_{\text {in, } l}-q_{\text {out }, l}$, with $q_{\text {out }, l}=K_{l} h_{l}$, where $h_{l}$ is the level, $K_{l}$ is a constant factor characterizing the outflow, $q_{\text {in }, l}$ is the inflow, and $q_{\text {out }, l}$ is the outflow of the $l^{\text {th }}$ tank. There is a constant available resource given by $Q=30$. Each inflow $q_{i n, l}$ is controlled by a valve commanded by a control signal $x_{l}$, i.e., $q_{i n, l}=Q x_{l}$, with $0 \leq x_{l} \leq 1$. It is assumed that there is a local controller at each valve that guarantees the desired flow given by $x_{l}$. The limited resource establishes a constraint over all inflows, i.e., $\sum_{i=1}^{N} q_{i n, l}=Q$. This constraint leads to the condition $\sum_{i=1}^{N} Q x_{l}=Q$, and consequently, $\mathbf{x} \in \Delta$. This condition is also satisfied in the partitioned system if the initial condition of the proportion of agents satisfies $\sum_{j \in \mathcal{P}_{i}} \mathbb{1}_{N_{i}^{j}}^{\top} \mathbf{x}_{i}^{j}(0)=1$, for the initial topology $i \in \mathcal{T}$, following Proposition 1 .

\section{RESULTS}

As a reference to analyze the performance of the proposed control strategy, results for the centralized case are also presented, i.e., the case with the unique topology given by a complete graph, or with the grand coalition (see Figure 1b)).

For the presented example, $\Delta t=0.1 \mathrm{~s}$. The Shapley value is computed in function of the error costs (7). It is important to mention that initial condition for each tank level has been established as a random value within the interval $[0,1]$. The control objectives are given by: i) maintain all the tank levels at the same maximum value; and ii) determine the fair economical costs for the players, then more KPIs are defined to evaluate the performance. For instance, it is of interest to analyze the error of each tank level with respect to the average levels of the entire system, i.e., $K P I_{\text {error }, l}=\int_{t_{0}}^{t_{f}}\left(h_{l}-\frac{1}{N} \mathbf{h}^{\top} \mathbb{1}_{N}\right) d t, l \in \mathcal{V}$, where $\mathbf{h}=\left[\begin{array}{lll}h_{1} & \cdots & h_{N}\end{array}\right]^{\uparrow}$. Then, there is a KPI for the whole system in function of the mentioned error levels, i.e., $K P I_{\text {error }}=\sum_{l=1}^{N} K P I_{\text {error }, l}$. Finally, it is defined the settling time with criterion of the $5 \%$ as a KPI, i.e., $K P I_{\text {settling }}=\min _{t}\{t: \forall \tilde{t} \geq t, \underline{\mathbf{h}} \leq \mathbf{h}(\tilde{t}) \leq \overline{\mathbf{h}}\}$, where $\underline{\mathbf{h}}=0.95 \mathbf{h}^{*}, \overline{\mathbf{h}}=1.05 \mathbf{h}^{*}$, and $\mathbf{h}^{*}$ being the equilibrium point of the system.

Figure 2 shows the evolution of the tank levels for three different cases (coalitions of 2 players, coalitions of 3 players and the grand coalition), and it is also shown the gap that determines the settling time. The topology in the system is determined every $\Delta t$, Figure 3 shows some different topologies obtained based on the Shapley value at five time instants. In Figure 3, it can be seen how topologies vary dynamically with coalitions of two and three players. In Figure 2, it can be seen that the first control objective is met for all the cases. The second control objective is determined by the Shapley value as presented in Table II.

TABLE II

FAIR ECONOMICAL COSTS FOR EACH PLAYER.

\begin{tabular}{c||cc}
\multicolumn{1}{c||}{ Player } & \multicolumn{2}{c}{ Fair costs according Shapley value } \\
$l \in \mathcal{V}$ & $\left(\Phi(l, k) K P I_{\text {links }}\right) /\left(\sum_{r=1}^{N} \Phi(r, k)\right)$ \\
\hline \hline & Coalitions 2 Players & Coalitions 3 Players \\
1 & 68.5457 & 163.2923 \\
2 & 88.3214 & 176.9323 \\
3 & 66.0268 & 296.4736 \\
4 & 85.9647 & 202.0730 \\
5 & 122.4891 & 172.0995 \\
6 & 107.3169 & 223.4468 \\
7 & 71.7682 & 197.5349 \\
8 & 147.2266 & 248.4602 \\
9 & 130.3143 & 237.5863 \\
10 & 124.9356 & 145.3167 \\
11 & 106.6134 & 169.3707 \\
12 & 80.4768 & 167.4130 \\
\hline Total & 1200 & 2400 \\
\hline
\end{tabular}




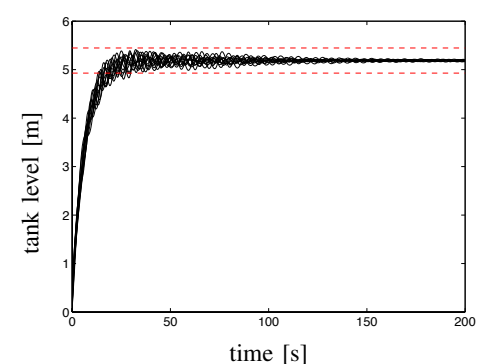

a)

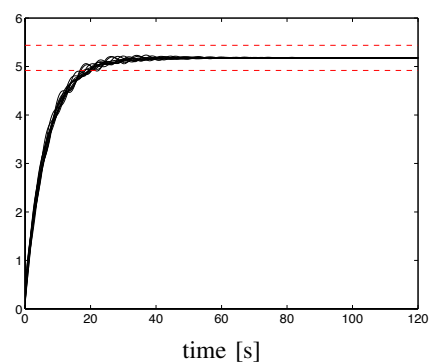

b)

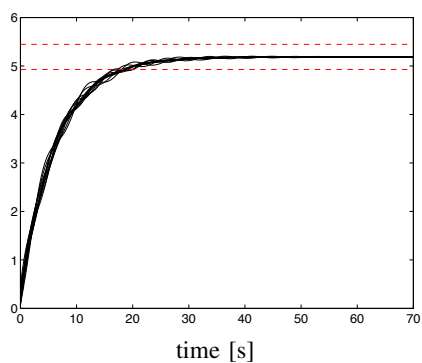

c)

Fig. 2. Evolution of tank levels for three cases. a) coalitions of 2 players, b) coalition of three players, and c) grand coalition $N$ players (full information). Initial condition for each tank level has been determined by a random value in the interval $[0,1]$.
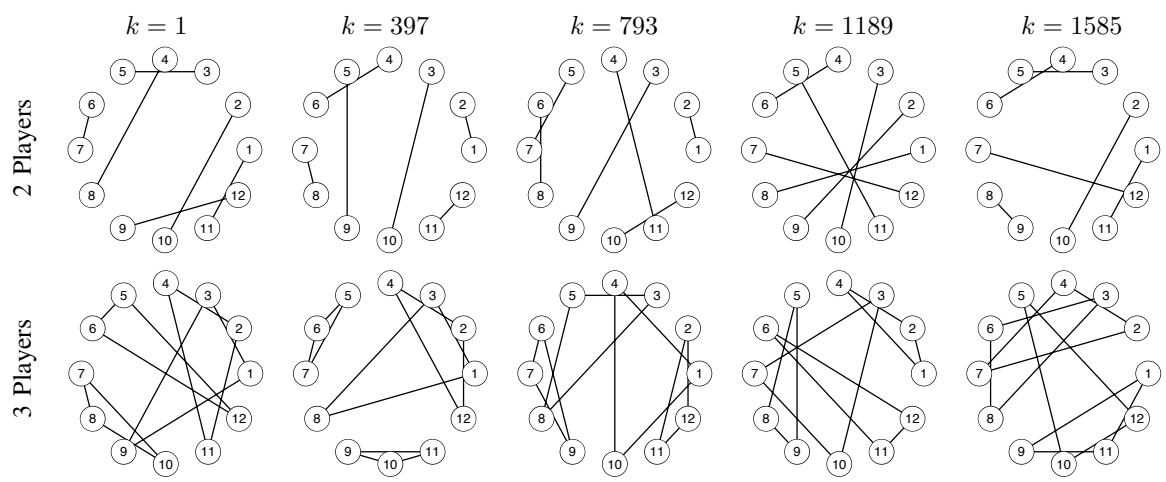

Fig. 3. Evolution of the topology graphs given by coalitions of two and three players (i.e., $g=2$, and $g=3$ ), for five different iterations, i.e., $k=1, \ldots, 1585$.

Finally, the comparison of the three cases with the three proposed KPI is presented in Table III. In these results, it can be seen the proper performance of the proposed methodology with respect to the centralized case with full information given by the grand coalition. Economical costs are reduced significantly by sacrifying transitory errors and settling time.

TABLE III

CONTROL PERFORMANCE FOR DIFFERENT TOPOLOGIES.

\begin{tabular}{c||ccc}
$\begin{array}{c}\text { Players per } \\
\text { coalitions } g\end{array}$ & $\begin{array}{c}\text { Communication } \\
K P I_{\text {links }}\end{array}$ & $\begin{array}{c}\text { Error } \\
K P I_{\text {error }}\end{array}$ & $\begin{array}{c}\text { Settle time } \\
K P I_{\text {settling }}\end{array}$ \\
\hline \hline 2 players & 1200 & 94.7748 & 29.76 \\
3 players & 2400 & 31.9961 & 22.51 \\
$N$ players & 13200 & 19.6141 & 20.14 \\
\hline
\end{tabular}

\section{CONCLUSIONS}

A multi-objective control problem that combines both cooperation and competition has been presented. The first control objective has been associated to a resource allocation problem, where it has been desired to achieve a maximum and equal level for all the tanks, and the second control objective has been to designed determine a "fair" distribution of communication costs. It has been proposed to use a noncooperative-game approach to solve the former objective, whereas a cooperative-game approach has been proposed to solve the latter objective. Moreover, an alternative way to compute the Shapley value for the selected characteristic function has been presented in order to mitigate the high computational burden issue.

\section{REFERENCES}

[1] J. Barreiro-Gomez, N. Quijano, and C. Ocampo-Martinez. Distributed constrained optimization based on population dynamics. In Proceedings of the IEEE Conference on Decision and Control (CDC), pages 4260-4265, Los Angeles - USA, 2014.
[2] R. Gopalakrishnan, J. Marden, and A. Wierman. Characterizing distribution rules for cost sharing games. In 5th International Conference on Network Games, Control and Optimization (NetGCooP), pages 1-4, Paris, France, 2011.

[3] M. Jilg and O. Stursberg. Optimized distributed control and topology design for hierarchically interconnected systems. In Proceedings of the 2013 European Control Conference, pages 4340-4346, Zurich, Switzerland, 2013.

[4] M. A. Khan, H. Tembine, and A. V Vasilakos. Evolutionary coalitional games: design and challenges in wireless networks. Wireless Communications, IEEE, 19(2):50-56, 2012.

[5] S. Li, Y. Zhang, and Q. Zhu. Nash-optimization enhanced distributed model predictive control applied to the shell benchmark problem. Inf. Sci. Inf. Comput. Sci., 170(2-4):329-349, 2005.

[6] J.M. Maestre, D. Muñoz de la Peña, A. Jiménez Losada, E. Algaba, and E.F. Camacho. A coalitional control scheme with applications to cooperative game theory. Optimal Control Applications and Methods, 35:592-608, 2014.

[7] F. J. Muros Ponce, J. M. Maestre, E. Algaba, C. Ocampo-Martinez, and E. F. Camacho. An application of the Shapley value to perform system partitioning. In Proceedings of the American Control Conference (ACC), pages 2143-2148, Chicago, USA, 2015.

[8] R. B. Myerson. Game Theory. Analysis of Conflict. Hardvard University Press, 1997.

[9] J. Nash. Equilibrium points in n-person games. Proc. of the National Academy of Sciences, 36:48-49, 1950.

[10] G. Obando, A. Pantoja, and N. Quijano. Building temperature control based on population dynamics. IEEE Transactions on Control Systems Technology., 22(1):404-412, 2014.

[11] E. Ramirez-Llanos and N. Quijano. A population dynamics approach for the water distribution problem. International Journal of Control, 83(9):1947-1964, 2010.

[12] S. Riverso, M. Farina, and G. Ferrari-Trecate. Plug-and-play decentralized model predictive control for linear systems. IEEE Transactions on Automatic Control, 58(10):2608-2614, 2013.

[13] W. H. Sandholm. Population games and evolutionary dynamics. Cambridge, Mass. MIT Press, 2010.

[14] L.S. Shapley. A value for n-person games. Annals of Math. Studies, 28:307-317, 1953.

[15] H. Tembine, E. Altman, R. El-Azouzi, and Y. Hayel. Evolutionary games in wireless networks. IEEE Transactions on Systems, Man, and Cybernetics, Part B: Cybernetics, 40(3):634-646, 2010. 